\title{
Quercetin administration reduces the pro-inflammatory cytokines expression in cirrhotic rats
}

\author{
J. M. Culebras ${ }^{1,2}$, J. Tieppo ${ }^{3}$, M. J. Cuevas ${ }^{1}$, R. Vercelino ${ }^{3}$, J. González-Gallego $^{1}$, N. Marroni ${ }^{3}$ \\ and M. J. Tuñón ${ }^{1}$ \\ ${ }^{1}$ CIBERehd, Institute of Biomedicina of the University of Leon, Spain, ${ }^{2}$ Hospital of León, Spain and ${ }^{3}$ Laboratorio \\ de Hepatologia Experimental Fisiologia HCPA/UFRGS, Porto Alegre, Brazil
}

Hepatic inflammation is an important pathophysiological process that precedes the development of liver cirrhosis ${ }^{(1)}$. Flavonoids are lowmolecular weight compounds found in citrus fruits, olive oil, tea, red wine, seeds and several medicinal plants. Anti-inflammatory properties of these compounds have been broadly studied ${ }^{(2)}$. Quercetin is the major flavonoid found in human diet. A number of beneficial effects of quercetin on human health have been also shown and some studies have indicated an important role for quercetin in several diseases, including liver cirrhosis ${ }^{(3)}$.

This study aims to elucidate the effects of quercetin on the gene expression of pro-inflammatory factors, including transforming growth factor-beta (TGF- $\alpha$ ), tumour necrosis factor-alpha (TNF- $\alpha$ ) and interleukin-6 (IL-6), in an animal model of liver cirrhosis.

Rats were divided into four groups: animals submitted to common bile duct ligation (CBDL), sham (animals submitted to simulate CBDL), quercetin-treated sham and quercetin-treated CBDL. Quercetin (50 mg/kg) was administered for 2 weeks starting on day 14 after surgery. On the 28th day, blood was collected for the analysis of aspartate aminotransferase (AST), alanine aminotransferase (ALT), alkaline phosphatase (ALP). Analysis of TGF- $\alpha$, TNF- $\alpha$ and IL-6 mRNA expression was performed in liver tissue by quantitative real time RT-PCR.

Blood AST, ALT and ALP activities and mRNA levels of pro-inflammatory cytokines were significantly higher in untreated CBDL rats compared to sham rats. The flavonoid quercetin significantly lowered serum ALT, AST and ALP activities (indicators of hepatocellular damage) and consistently caused a reduction of mRNA levels of TGF- $\alpha$, TNF- $\alpha$ and IL- 6 , in treated cirrhotic animals compared with untreated CBDL rats.

Thus, quercetin treatment, initiated following the establishment of liver injury in cirrhotic animals, has a clear anti-inflammatory effect in the subsequent severity of the CBDL-related inflammation. This result supports that trials of quercetin may be reasonable in patients with established liver disease and inflammation.

1. Friedman SL (2004) Nat Clin Pract Gastroenterol Hepatol 1, 98-105.

2. Rotelli AE, Aguilar CF \& Pelzer LE (2009) Eur Biophys J 38, 865-871. doi: 10.1007/s00249-009-0453-x

3. Peres W, Tuñón MJ, Collado PS et al. (2000) J Hepatol 33, 742-750. 